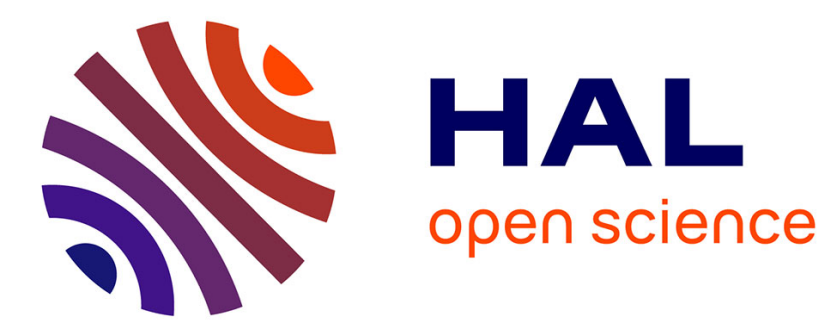

\title{
Adaptive foraging does not always lead to more complex food webs
}

Luděk Berec, Jan Eisner, Vlastimil Křivan

\section{To cite this version:}

Luděk Berec, Jan Eisner, Vlastimil Křivan. Adaptive foraging does not always lead to more complex food webs. Journal of Theoretical Biology, 2010, 266 (2), pp.211. 10.1016/j.jtbi.2010.06.034 . hal00614190

\section{HAL Id: hal-00614190 \\ https://hal.science/hal-00614190}

Submitted on 10 Aug 2011

HAL is a multi-disciplinary open access archive for the deposit and dissemination of scientific research documents, whether they are published or not. The documents may come from teaching and research institutions in France or abroad, or from public or private research centers.
L'archive ouverte pluridisciplinaire HAL, est destinée au dépôt et à la diffusion de documents scientifiques de niveau recherche, publiés ou non, émanant des établissements d'enseignement et de recherche français ou étrangers, des laboratoires publics ou privés. 


\section{Author's Accepted Manuscript}

Adaptive foraging does not always lead to more complex food webs

Luděk Berec, Jan Eisner, Vlastimil Křivan

PII: S0022-5193(10)00325-5

DOI: doi:10.1016/j.jtbi.2010.06.034

Reference: YJTBI 6054

To appear in: $\quad$ Journal of Theoretical Biology

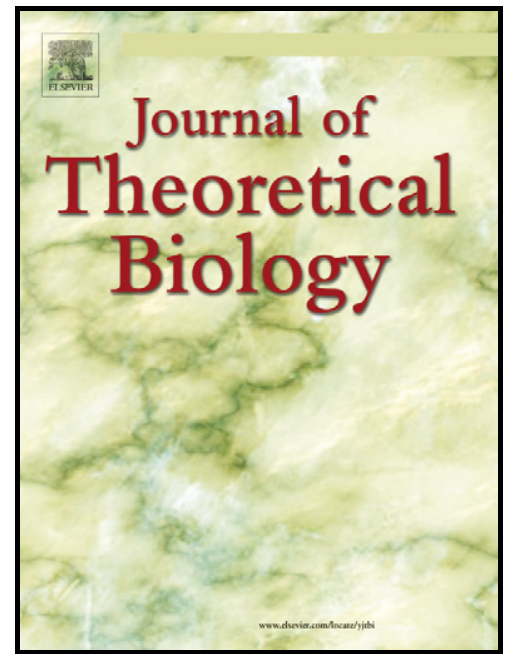

www.elsevier.com/locate/yjtb

Received date: $\quad 30$ October 2009

Revised date: $\quad 2$ June 2010

Accepted date: $\quad 24$ June 2010

Cite this article as: Luděk Berec, Jan Eisner and Vlastimil Křivan, Adaptive foraging does not always lead to more complex food webs, Journal of Theoretical Biology, doi:10.1016/j.jtbi.2010.06.034

This is a PDF file of an unedited manuscript that has been accepted for publication. As a service to our customers we are providing this early version of the manuscript. The manuscript will undergo copyediting, typesetting, and review of the resulting galley proof before it is published in its final citable form. Please note that during the production process errors may be discovered which could affect the content, and all legal disclaimers that apply to the journal pertain. 


\section{Adaptive foraging does not always lead to more complex food webs}

2

3

4

5

6

Luděk Berec*, Jan Eisner, Vlastimil Křivan

Department of Theoretical Ecology, Institute of Entomology, Biology Centre ASCR, and Institute of Mathematics and Biomathematics, Faculty of Science, University of South Bohemia, Branišovská 31, 37005 České Budějovice, Czech Republic

${ }^{*}$ Corresponding author: Luděk Berec, Department of Theoretical Ecology, Institute of Entomology, Biology Centre ASCR, Branišovská 31, 37005 České Budějovice, Czech Republic, e-mail: berec@entu.cas.cz, phone: +420-387775293, fax: +420-385310354

E-mail addresses: berec@entu.cas.cz (L. Berec), eisner@math.cas.cz (J. Eisner), vlastimil.krivan@gmail.com (V. Krivan) 
1 Abstract: Recent modeling studies exploring the effect of consumers' adaptivity in diet composition on food web complexity invariably suggest that adaptivity in foraging decisions of consumers makes food webs more complex. That is, it allows for survival of a higher number of species when compared with non-adaptive food webs. Population-dynamical models in these studies share two features: parameters are chosen uniformly for all species, i.e., they are species-independent, and adaptive foraging is described by the search image model. In this article, we relax both these assumptions. Specifically, we allow parameters to vary among the species and consider the diet choice model as an alternative model of adaptive foraging. Our analysis leads to three important predictions. First, for species-independent parameter values for which the search image model demonstrates a significant effect of adaptive foraging on food web complexity, the diet choice model produces no such effect. Second, the effect of adaptive foraging through the search image model attenuates when parameter values cease to be speciesindependent. Finally, for the diet choice model we observe no (significant) effect of adaptive foraging on food web complexity. All these observations suggest that adaptive foraging does not always lead to more complex food webs. As a corollary, future studies of food web dynamics should pay careful attention to the choice of type of adaptive foraging model as well as of parameter values.

Keywords: diet choice, population dynamics, niche model, search image, replicator dynamics 


\section{Introduction}

Presumably the fundamental question of ecology is how species diversity is maintained in nature or, alternatively, why species communities of different complexity are found in different ecosystems such as arid zones, tropical forests, coastal waters or open ocean. Despite some 100 years of research, since Eugenius Warming and Ernst Haeckel founded ecology as a scientific discipline (Goodland 1975), we are still far from a complete answer. In fact, due to complexity of this question, ecologists have focused on simpler, better accessible problems. These include questions such as how are species communities structured (Dunne 2006, and references therein), or what ecological mechanisms tend to stabilize community dynamics (Kondoh 2003a; Martinez et al. 2006).

In the quest for answers to these questions, ecologists work with the concept of food webs, which are complex ecological networks describing, in the simplest case, who eats whom in a species community. As it is difficult to deal with highly complex food webs, researchers have sometimes lumped functionally similar species together (e.g., Yodzis and Winemiller 1999) and/or thought of food webs as collections of simple building blocks, called community modules in ecology (Holt 1995) and motifs in the gene networks literature (e.g., Milo et al. 2002). Mathematical analysis of such community modules (including food chain, exploitative competition, apparent competition, omnivory and others) is relatively straightforward and has revealed important effects of their structure on population dynamics, through both direct and indirect interactions (Holt 1995). This suggests that food web structure is one of the major determinants of population dynamics. Conversely, population dynamics shape food web structure through species invasions and/or extinctions. Altogether, there is a complex feedback between food web structure and population dynamics, and we need to consider both to understand the main mechanisms that shape ecological communities.

Since the pioneering work of Robert May on the relationship between food web complexity and stability (May 1972), researchers have become interested in ecological mechanisms that promote species diversity. Here community modules enter the stage again: potential mechanisms recruit from those that tend to stabilize dynamics of module-forming populations. These 
include adaptivity of consumers with respect to their diet composition (e.g., Gleeson and Wilson 1986; Fryxell and Lundberg 1994; Fryxell and Lundberg 1997; Krrivan 1997; Křivan and Sikder 1999), type III functional response (e.g., Murdoch and Oaten 1975; Williams and Martinez 2004), degree of immigration (Bastolla et al. 2001), and intraspecific interference in consumers (Ruxton 1995; Huisman and De Boer 1997). Studies exploring the impact of these mechanisms on food web complexity almost invariably adopt the "top-down approach": an "initial" food web structure is generated first and then population dynamics are run on this food web. After the dynamics attain an attractor, the "eventual" food web structure is recorded. A feature common to this approach is that initial food webs virtually always collapse, with only a proportion of species from those initially present that eventually persist (e.g., Bastolla et al. 2001; Kondoh 2003a; Brose et al. 2003, but see Williams and Martinez 2004).

The top-down approach has been repeatedly used to study effects of consumers' adaptivity in diet composition on food web complexity (Brose et al. 2003; Kondoh 2003a; Kondoh 2003b; Kondoh 2006; Garcia-Domingo and Saldana 2007; Uchida and Drossel 2007). Results of these works invariably suggest that adaptivity in foraging decisions of consumers increases food web complexity (i.e., the number or proportion of species surviving in the eventual food webs) when compared with food webs in which consumers have fixed (i.e., non-adaptive) preferences for their resources. This observation is also one of the major conclusions put forth in the recent review on the consequences of adaptive foraging in food webs (Loeuille 2010). However, all these works assumed that most, if not all, parameters determining population dynamics are the same for all species in the food web, i.e., there is no between-species variability in parameter values. In addition, they considered just one type of adaptive foraging that we refer to as the search image model below: consumers form a search image for and hence put most of their effort into the most abundant (or more generally the most profitable) resource.

In this article, we extend the previous analyses by allowing parameters determining population dynamics to vary among the species in the food web, and using the diet choice model (Charnov 1976) as an alternative model of adaptive foraging. The diet choice model assumes that upon each encounter with a resource item, a consumer decides whether it will attack that item or not. Therefore, we explore how adaptivity in foraging decisions of consumers affects food web 
complexity, whether these predictions are sensitive to the choice of foraging model, and how does this complexity depend on variability in model parameters. In other words, our major aim here is to study effects of the diet choice model and compare them with the results established in the literature for the search image model. We also want to study robustness of predictions of both these models with respect to parameter values.

\section{Methods}

\section{Simulation procedure}

To examine the impact of adaptive foraging on food web dynamics, we start with generating a topological food web structure (i.e., nodes representing populations of different species and links connecting these nodes that represent consumer-resource interactions). Then, we define a population-dynamical model on this structure, including a type of adaptive foraging. Finally, we parameterize the model and run it for a sufficient period of time to allow system trajectories to attain an attractor. The variable of prime interest to us is the proportion of species still present in the food web at the end of that period. This sequence of events represents one simulation run. As the procedures used to generate the food web structure, parameter values and initial population densities all contain an element of chance (see below), one scenario consists of a number of simulation runs from which the mean and a measure of variability of the variable of interest is calculated.

\section{Food web structure}

We use the niche model (Williams and Martinez 2000) to generate the (initial) food web structure. The input parameters of this model are the number of species $S$ and the connectance $C$ (mean number of links per species). The niche model assigns a random value drawn uniformly from the interval $[0,1]$ to each species. This value is called the species' niche value, $n_{i}$. Each 
species consumes all species within a range of niche values $\left[c_{i}-r_{i} / 2, c_{i}+r_{i} / 2\right]$ where the width of this range $r_{i}$ is randomly assigned using a beta distribution and its center $c_{i}$ is drawn uniformly from the interval $\left[r_{i} / 2, n_{i}\right]$ (or $\left[r_{i} / 2,1-r_{i} / 2\right]$ if $n_{i}>1-r_{i} / 2$ ). The beta distribution is parameterized so that the actual connectance of generated food webs lies close to the prescribed connectance $C$ (Williams and Martinez 2000); see also Allesina et al. (2008) for a subtle correction in the beta distribution parameterization that is more precise for small numbers of species $S$ - for $S=30$ that we use in this paper the difference is insignificant. Not all generated food webs are accepted for further processing, however. The webs that contain an isolated species (that is, species with neither incoming nor outgoing links) and webs whose actual connectance differs from the prescribed one by more than $3 \%$ are discarded.

\section{Population dynamics}

We distinguish basal species, i.e., species that have no resource (no incoming links), and nonbasal species consuming a resource (at least one incoming link). We assume that all basal species grow logistically in the absence of consumers, and that all non-basal species die out exponentially in the absence of resources. To model consumption we use the Holling type II functional response for multiple resources. The rate of change of density $x_{i}$ of population $i$ $(i=1, \ldots, S)$ is therefore

$$
\frac{d x_{i}}{d t}=\left(r_{i}-s_{i} x_{i}\right) x_{i}-d_{i} x_{i}+\frac{\sum_{j=1}^{S} p_{j i} a_{j i} e_{j i} \lambda_{j i} x_{j}}{1+\sum_{j=1}^{S} p_{j i} a_{j i} h_{j i} \lambda_{j i} x_{j}} x_{i}-\sum_{j=1}^{S} \frac{p_{i j} a_{i j} \lambda_{i j} x_{i}}{1+\sum_{k=1}^{S} p_{k j} a_{k j} h_{k j} \lambda_{k j} x_{k}} x_{j}
$$

The matrix $A=\left(a_{i j}\right)$ is the interaction matrix generated by the niche model $\left(a_{i j}=1\right.$ if species $j$ consumes species $i$ and $a_{i j}=0$ otherwise) and the matrix $P=\left(p_{i j}\right)$ is the matrix of preferences determined by an adaptive foraging model (see below). Hence, the matrix $A$ sets the structural constraints on the food web (e.g., to avoid herbivores to feed on carnivores etc.). Feeding preferences modeled by the matrix $P$ then describe behavioral flexibility with respect to each of the resource species for consumers that have at least two links to potential resources; $p_{i j}=1$ if $i$ is the only resource of consumer $j$. The remaining parameters are explained in Table 1. By definition, basal species have $d_{i}=0$ and non-basal species have $r_{i}=s_{i}=0$. We remark that 


\section{Adaptive foraging}

population dynamics (1) assume an environment where all consumers and all resources mix homogeneously. Thus, this model does not describe patchy environments in which each of the patches contains one or a few resources only.

Adaptive foraging prescribes how consumers' preferences for available resources (i.e., diet composition) respond to the density of these resources so that the consumers' per capita food intake rate is maximized. In this article, we consider two models of adaptive foraging. The first builds on the switching model sensu Murdoch (1969). One of the mechanisms that can lead to such switching behavior is formation of search images sensu Tinbergen (1960) and we will call this model the search image model; see also Bond (1983). The second model we use is the diet choice model (Charnov 1976). Whereas the search image model determines how a consumer distributes its preferences between resources or, alternatively, what proportion of its foraging time the consumer spends feeding on each resource (Abrams 1999; Kondoh 2003a), the diet choice model assumes that the consumer has to decide upon each encounter with a resource item on whether it will attack that item or not (Charnov 1976; Stephens and Krebs 1986; Křivan 1996; Houston and McNamara 1999; Berec and Křivan 2000; Berec et al. 2003). As a consequence, in the search image model, $p_{i j}$ represents the proportion of foraging time consumer $j$ feeds on resource $i$. On the other hand, in the diet choice model, $p_{i j}$ represents the probability that consumer $j$ attacks resource $i$ upon encounter. Therefore, for each consumer species, $0 \leq p_{i j} \leq 1$ in both models; whereas $\sum_{i=1}^{S} p_{i j}=1$ in the search image model, there is no such restriction in the diet choice model.

\section{The search image model}

This model of adaptive foraging assumes that consumers form a search image for the most abundant or, more generally, the most profitable resource. The idea is that experience of the consumer with a less abundant or less profitable resource is so limited that they do not consider 
where

$$
\frac{d p_{i j}}{d t}=\nu p_{i j} a_{i j}\left(a_{i j} e_{i j} \lambda_{i j} f_{i j}-\sum_{k=1}^{S} p_{k j} a_{k j} e_{k j} \lambda_{k j} f_{k j}\right)
$$

$$
f_{i j}=\frac{x_{i}}{1+\sum_{k=1}^{S} p_{k j} a_{k j} h_{k j} \lambda_{k j} x_{k}}
$$

The parameter $\nu$ here represents the adaptation rate. According to this model, feeding preferences for which the per capita food intake rate is above the average get stronger while those consumer-resource links that provide a below average food intake rate get weaker. This nicely describes the idea of forming a search image. We remark that if a link exists $\left(a_{i j}=1\right)$, but initially the preference of consumer $j$ for resource $i$ is zero $\left(p_{i j}=0\right)$, then this preference will never become positive. To avoid this, all initial preferences for admissible links specified by the connectivity matrix $A$ are set positive. Thus, the number of links in a simulation run can only decrease, because some links will die out. In addition, if initially a preference is very low, it can take relatively long time for this preference to increase.

The search image model (2) has been frequently used in studies of food web dynamics (Kondoh 2003a; Kondoh 2003b; Brose et al. 2003; Kondoh 2006). We note, however, that the models used in Brose et al. (2003) and Kondoh (2006) contain a typo as the first term in the parentheses is $p_{i j} a_{i j} e_{i j} \lambda_{i j} f_{i j}$ there. If this were so, the whole expression in the parentheses would be negative and any preference $p_{i j}$ could thus only decrease. As a consequence, the constraint $\sum_{i=1}^{S} p_{i j}=1$ could not be guaranteed.

\section{The diet choice model}

The diet choice model (Charnov 1976) determines the probability with which a consumer attacks a resource item upon encounter so that its long-term per capita food intake rate is maximized. This probability can be calculated as follows (Stephens and Krebs 1986, for an alternative derivation see Houston and McNamara 1999). First, all resource species of consumer 
$1 j$ (i.e., all species $i$ for which $a_{i j}=1$ ) are ranked in the descending order according to their 2 "profitability" $e_{i j} / h_{i j}$. Second, the most profitable resource species is added to the consumer's 3 optimal diet, and then the other species are added in the order of their (decreasing) profitability 4 until

$$
\frac{\sum_{i=1}^{k} e_{i j} \lambda_{i j} x_{i}}{1+\sum_{i=1}^{k} h_{i j} \lambda_{i j} x_{i}}>\frac{e_{(k+1) j}}{h_{(k+1) j}}
$$

where $k$ is the number of resources already in the diet. This procedure is repeated for all nonbasal species and a matrix $P^{\mathrm{opt}}$ is eventually composed so that $p_{i j}^{\mathrm{opt}}=1$ if species $i$ is in the optimal diet of species $j$, and $p_{i j}^{\mathrm{opt}}=0$ otherwise.

The optimal preferences $p_{i j}^{\text {opt }}$ depend on the actual population densities. If adaptive foragers adjust their diet instantaneously to the actual population densities, then $p_{i j}=p_{i j}^{\text {opt }}$ in model (1). Such an instantaneous adjustment is not very realistic and we assume that preferences $p_{i j}$ change dynamically as

$$
\frac{d p_{i j}}{d t} \in \nu\left(p_{i j}^{\mathrm{opt}}(x)-p_{i j}\right)
$$

where $\nu$ again represents the adaptation rate - the rate at which the actual diet attains the optimal one and $x=\left(x_{1}, \ldots, x_{S}\right)$ is the vector of population densities. High values of $\nu$ mean that consumer preferences keep track of changes in population densities very quickly, while low values of $\nu$ describe sub-optimal foraging. The preference dynamics (5) are called the best response dynamics (e.g., Gilboa and Matsui 1991; Hofbauer 1995; Hofbauer and Sigmund 1998; Křivan et al. 2008). A possible mechanism that generates model (5) assumes that a small proportion of consumers revise their preferences to the present best choice in each small time interval. We note that optimal preferences $p_{i j}^{\text {opt }}$ are not uniquely defined for some non-generic population densities (Křivan 1996) - that is why we use symbol $\in$ instead of $=$ in expression (5).

Although the diet choice model was used to generate food web topology (Beckerman et al. 2006; Petchey et al. 2008), to our knowledge it has not been used in relation to dynamics on complex food webs yet (but see an analysis carried out by Uchida et al. (2007) who focused on a food web module consisting of two predators, two prey and one resource with some additional constraints on feeding preferences). 


\section{Scenarios, parameter values and simulation runs}

Each baseline scenario that we consider comprises an (initial) number of species $S=30$, food web connectance $C$ (in the range 0.1-0.3 with step size 0.05 ), set of parameter values and initial population densities (two parameter sets, specified in Table 1), proportion of consumers feeding on two or more resources that are adaptive (none or all), adaptive foraging model (the search image model or the diet choice model), and adaptation rate $\nu($ slow $=0.25$ and fast $=4)$. We thus have 80 different baseline scenarios in total. Each scenario consists of 50 simulation runs for which the proportion of species surviving at the end is recorded. The period of time over which simulations are run is 2000 time units, sufficiently large for persistent populations to attain an attractor (steady state or limit cycle). The mean and a measure of variability are calculated for these recorded data, with the latter allowing for direct visual assessment of statistical significance of differences between any two scenarios (Andrews et al. 1980).

For each simulation run, parameter values either do not vary among the species (i.e., are speciesindependent) or do vary among the species. We consider two baseline sets of parameter values (Table 1). The parameter set 1 is intended to mimic as closely as possible the values used by Brose et al. (2003) and the results of their model, namely, the impact of adaptive foraging through the search image model on the proportion of species that survive from those forming the initial food web. The parameter set 2 is a "randomized" version of the parameter set 1 for which fixed parameter values of the set 1 were used as midpoints of parameter ranges from which actual values were then randomly (uniformly) chosen and thus a variability of parameters over species ensured; this is a standard procedure within the top-down approach (Brose et al. 2003; Kondoh 2003a; Kondoh 2003b; Martinez et al. 2006).

Regarding the initial values of consumer preferences $p_{i j}$, we set $p_{i j}=1$ for the diet choice model as soon as $a_{i j}=1$ and $p_{i j}=0$ otherwise. For the search image model, we set $p_{i j}=$ $1 / \sum_{i=1}^{S} a_{i j}$ for consumer $j$ as soon as $a_{i j}=1$ and $p_{i j}=0$ otherwise. That is, consumers initially attack all resource species they encounter in the diet choice model, and have equal (proportional) preference for any resource species in the search image model. 
1 All models were solved numerically, using the Embedded Runge-Kutta-Fehlberg $(4,5)$ method

of the GNU Scientific Library (GSL), a numerical library for $\mathrm{C}$ and $\mathrm{C}++$ programmers (http://www.gnu.org/software/gsl/). We consider a species extinct once its density falls below a small threshold value, arbitrarily set to $\epsilon=10^{-6}$ (using $\epsilon=10^{-10}$ did not produce qualitatively different results). Contrary to many previous works, however, we do not evaluate species persistence at the end of the simulation period. Rather, we remove a species from the food web as soon as its density drops below the threshold value. Although in our study the evaluation of species persistence at the end of the simulation period did not produce qualitatively different results, we believe that the approach consisting of the continuous removal of "extinct" species is generally more robust. For example, in the latter approach, once a species density falls below the threshold value the species cannot recover; it may thus account for demographic stochasticity.

\section{Results}

The simulation results for the baseline scenarios are summarized in Fig. 1. In line with the results of many previous studies and regardless of the scenario, the proportion of surviving species declines with the increasing (initial) food web connectance. In other words, the more "wired" is the initial food web, the more species extinctions we observe in each simulation run. This is in part due to the decreasing number of basal species and hence larger energy limitation of food webs generated by the niche model as the (initial) connectance $C$ increases. Indeed, the mean (initial) number of basal species in a sample of 10,000 generated food webs is 8.08 , 5.93, 4.57, 3.58 and 2.83 for $C=0.1,0.15,0.2,0.25$ and 0.3 , respectively, which gives the (initial) proportion of basal species among all species equal to $0.27,0.2,0.15,0.12$ and 0.09 , respectively. Of the baseline scenarios, the highest proportion of persisting species occurs for the search image model with the parameter set 1 (the same parameters for all species), adaptivity switched on and the lowest examined (initial) connectance $(C=0.1)$ (Fig. 1).

The search image model and the diet choice model produced entirely different results. For 
the search image model and the parameter set 1 (i.e., the same parameters for all species), a scenario close to that explored by Brose et al. (2003), the effect of adaptivity on the proportion of surviving species is the most pronounced (Fig. 1A, gray lines); our results thus confirm those of Brose et al. (2003). Contrary to that, adaptive foraging modeled by the diet choice model has no (significant) effect on the proportion of surviving species (Fig. 1A, black lines). The effect of adaptivity also declines if the search image model is used with the parameter set 2 (variability in parameter values among the species) instead of the parameter set 1 (Fig. 1B). The results for food webs with fast adaptation are analogous (Fig. 1C-D). Overall, these results suggest that the type of adaptive foraging model considerably affects food web complexity. Note that in general results for the two adaptive foraging models differ even if there is no adaptivity; this is due to the different choice of initial values of consumer preferences $p_{i j}$ in these models (see the "Methods" section).

Some additional parameter sets to those listed in Table 1 have been explored, with results analogous to those given above. Figure 2 shows how the proportion of surviving species varies with the consumer-resource encounter rate $\lambda$, the other parameters being those of the parameter set 1. For sufficiently low values of $\lambda$, consumers cannot feed enough to replace themselves and the only species that survive are basal species (note that for $\lambda \sim 1$, the diet choice model outperforms the search image one). At the other extreme of large values of $\lambda$, consumers overexploit their resources, some of basal species go extinct, and what remains is an impoverished food web with just a few basal and non-basal species (note that for $\lambda \geq 20$, the search image model without adaptivity performs better than the same model with adaptivity). In between these two extreme cases, the results do not vary qualitatively with $\lambda$. We remark here that simulations in Fig. 1 assume $\lambda=6$ for which the food webs are not limited by energy transfer.

Figure 3 shows the effects of adaptation rate $\nu$ on species persistence. The only situation in which we observe a qualitative change concerns the search image model with adaptivity and the parameter set 2 (solid gray line in Fig. 3B); whereas adaptivity in this case enhances food web complexity for $\nu \leq 4$, it appears to have a negative effect for $\nu \geq 6$.

Figure 4 shows qualitative character of population dynamics for $S=30, \nu=0.25$ and the 
parameter set 2. Majority of simulation runs produced fluctuating trajectories, irrespectively of the adaptive foraging model and adaptivity switched on or off (Fig. 4A). The effect of adaptivity on the extent of these fluctuations differs, though. Adaptivity through the diet choice model seems to affect both the average amplitude (Fig. 4B) and the maximum amplitude of these fluctuations (Fig. 4C) only slightly. On the other hand, adaptivity through the search image model tends to lead on average to larger fluctuations (Fig. 4C). The two models of adaptive foraging thus have contrasting effects on dynamics of the involved populations.

Garcia-Domingo and Saldana (2007) argued that the effect of food web complexity on species persistence should be quantified through relating the proportion of surviving species to the final food web connectance, rather than to the initial one as we and all virtually previous studies actually did. Through this methodological change, Garcia-Domingo and Saldana showed that some complexity-stability relationships that were positive when referring to the initial food web connectance turned out to be negative when referring to the final food web connectance. Although we do not address at all direction of these relationships in this paper, we tested for robustness (or not) of our results by replacing the initial connectance with the final one. Simulations show that qualitatively the effects of the two adaptive foraging models do not change - we still observe a significant effect of adaptivity through the search image model for parameter set 1 , reduced effect of adaptivity through the search image model for parameter set 2, and no (significant) effect of adaptivity through the diet choice model for any of the two parameter sets (Fig. 5).

\section{Discussion}

One of the major efforts in food web studies has been to come up with ecological mechanisms that increase food web persistence or produce sufficiently complex food webs in mathematical models of community dynamics. This is important both theoretically, to understand what keeps complex food webs together, and practically, to carry out further modeling experiments on such food webs, e.g., how robust or fragile are they to addition (invasion) or removal (extinction) of species. 
Recently, adaptive foraging has been considered one of such persistence-enhancing mechanisms and several studies appear to support this suggestion (Brose et al. 2003; Kondoh 2003a; Kondoh 2003b; Kondoh 2006; Garcia-Domingo and Saldana 2007; Uchida and Drossel 2007, see also a recent review paper by Loeuille 2010). However, these studies are limited by their assumptions. Specifically, all these studies were based on just one model of adaptive foraging (the search image model), and all assumed specific and species-independent parameter values, irrespectively of the species' character and trophic position (and hence assumed no between-species variability in their life histories).

Here we show that the role of adaptive foraging is not that straightforward as the previous analyses would suggest. Specifically, we carried out complementary simulations, using also the diet choice model of adaptive foraging and considering the possibility that diverse species can have diverse parameter values. The main message we would like to convey is that adaptivity per se may not necessarily enhance species persistence in complex food webs. In particular, we showed that in our simulations (i) the diet choice model of adaptive foraging does not enhance species persistence in complex food webs (Figs 1, 2, 3 and 5), (ii) the effect of adaptivity through the search image model on species persistence in complex food webs is highly sensitive to parameter values (Fig. 2), the degree they vary among the species (Fig. 1), and the adaptation rate (Fig. 3), (iii) there are parameter combinations for which the effects of both the search image model and the diet choice models on the proportion of surviving species are comparable (Figs 1D, 2 and 3B).

As our results show, the food web complexity is quite sensitive to both model structure and model parameters, irrespectively of whether we track the initial or final food web connectance as our independent variable (Garcia-Domingo and Saldana 2007), and it is therefore difficult if not impossible to come up with general predictions about the role of adaptive foraging on this complexity. Therefore, the assumptions should always be clearly spelled out and predictions obtained on the basis of simulations should not be extrapolated to other "similar" scenarios. In addition, specific assumptions used in modeling the impact of adaptive foraging on dynamics of complex food webs should always accompany results reported in other studies. 
A natural question here arises why the diet choice model does not (significantly) increase food web complexity while the search image model does (at least in some situations). We think this difference is related to the strength of interactions in the food web, which is different for the two foraging models. It was shown that weak interactions in food webs promote species coexistence (McCann et al. 1998). Also, analyses of simple two-resources-one-consumer food web modules suggest that the search image model promotes species coexistence when compared to the diet choice model because it relaxes apparent competition between the species more strongly than the diet choice model (Křivan and Sikder 1999; Křivan and Eisner 2003; Křivan and Eisner 2006). In the two-resources-one-consumer food web with the search image model this is because when a consumer preference for one resource increases, its feeding on the other resource decreases and this weakens apparent competition between the two resources. On the contrary, in the diet choice model the more profitable resource is never excluded from the consumer's diet so that the apparent competition between the two resource is never completely relaxed.

One might also be interested in why the positive effect of adaptivity through the search image model on species persistence decreases when parameters are chosen at random rather than being species-independent (cf. results in Fig. 1A-B for the parameter set 1 with results for a random choice of parameters from the set 2 shown in Fig. 1C-D). The species coexistence regions in the parameter space are defined by constraints (e.g., on existence and stability of an equilibrium) and the more species coexist in a given region, the more limiting constraints must be satisfied. Thus, in multi-dimensional models the region of parameter values where many species coexist is quite "small" - allowing for a variance in parameter values will cause some of these values to lie outside this region where some species will go extinct. We think this is the reason why there is a decrease in species persistence when results for the (fixed) parameter set 1 with the (variable) parameter set 2 are compared. Recall that the parameter set 1 mimics as closely as possible the values used by Brose et al. (2003) - see the Methods section for more details.

In summary, we show that differences in details of animal behavior can result in entirely different food webs and that the impact of adaptivity in consumers' diet composition on food web dynamics depends on the type of adaptivity considered and the degree of parameter variability 
1 across the involved species. Most importantly, we show that in many instances the effect of 2 adaptive foraging on food web complexity is negligible, and can sometimes be even negative.

3 Therefore, we are far from saying that adaptive foraging stabilizes food web dynamics, makes 4 food webs more complex or allows for survival of more species, as some recent studies suggest.

\section{Acknowledgments}

6 We acknowledge funding by the Institute of Entomology (Z50070508) and the Grant Agency 7 of the Academy of Sciences of the Czech Republic (IAA100070601).

\section{${ }_{8}$ References}

Abrams, P. A. 1999. The adaptive dynamics of consumer choice. American Naturalist 153:8397.

Allesina, S., D. Alonso and M. Pascual. 2008. A general model for food web structure. Science 320:658-661.

Andrews, H. P., R. D. Snee and M. H. Sarner. 1980. Graphical display of means. American Statistician 34:195-199.

Bastolla, U., M. Lassig, S. C. Manrubia and A. Valleriani. 2001. Diversity patterns from ecological models at dynamical equilibrium. Journal of Theoretical Biology 212:11-34.

Beckerman, A. P., O. L. Petchey and P. H. Warren. 2006. Foraging biology predicts food web complexity. Proceedings of the National Academy of Sciences of the USA 103:1374513749 .

Berec, L. and V. Křivan. 2000. A mechanistic model for partial preferences. Theoretical Population Biology 58:279-289. 
Berec, M., V. Křivan and L. Berec. 2003. Are great tits (Parus major) really optimal foragers? Canadian Journal of Zoology 81:780-788.

Bond, A. B. 1983. Visual search and selection of natural stimuli in the pigeon: the attention threshold hypothesis. Journal of Experimental Psychology: Animal Behavior Processes 9:292-306.

Brose, U., R. J. Williams and N. D. Martinez. 2003. Comment on "Foraging adaptation and the relationship between food web complexity and stability”. Science 301:918b.

Charnov, E. L. 1976. Optimal foraging: attack strategy of a mantid. American Naturalist 110:141-151.

Dunne, J. A. 2006. The network structure of food webs. Pages 27-86 in M. Pascual and J. A. Dunne, eds. Ecological networks: linking structure to dynamics in food webs. Oxford University Press, Oxford, UK.

Fryxell, J. M. and P. Lundberg. 1994. Diet choice and predator-prey dynamics. Evolutionary Ecology 8:407-421.

Fryxell, J. M. and P. Lundberg. 1997. Individual behavior and community dynamics. Chapman \& Hall, London, UK.

Garcia-Domingo, J. L. and J. Saldana. 2007. Food-web complexity emerging from ecological dynamics on adaptive networks. Journal of Theoretical Biology 247:819-826.

Gilboa, I. and A. Matsui. 1991. Social stability and equilibrium. Econometrica 59:859-867.

Gleeson, S. R. and D. S. Wilson. 1986. Equilibrium diet: optimal foraging and prey coexistence. Oikos 46:139-144.

Goodland, R. J. 1975. The tropical origin of ecology: Eugen Warming's jubilee. Oikos 26:240245.

Hofbauer, J. 1995. Stability for the best response dynamics. Mimeo, University of Vienna. 
Hofbauer, J. and K. Sigmund. 1988. The theory of evolution and dynamical systems. Cambridge University Press, Cambridge.

Hofbauer, J. and K. Sigmund. 1998. Evolutionary games and population dynamics. Cambridge University Press, Cambridge.

Holt, R. D. 1995. Community modules. Pages 333-350 in A. C. Gange and V. K. Brown, eds. Multitrophic interactions in terrestrial systems. Blackwell Science Ltd, London, UK.

Houston, A. I. and J. M. McNamara. 1999. Models of adaptive behaviour. Cambridge University Press, Cambridge, UK.

Huisman, G. and R. J. De Boer. 1997. A formal derivation of the "Beddington" functional response. Journal of Theoretical Biology 185:389-400.

Kondoh, M. 2003a. Foraging adaptation and the relationship between food web complexity and stability. Science 299:1388-1391.

Kondoh, M. 2003b. Response to comment on "Foraging adaptation and the relationship between food web complexity and stability". Science $301: 918$ c.

Kondoh, M. 2006. Does foraging adaptation create the positive complexity-stability relationship in realistic food-web structure? Journal of Theoretical Biology 238:646-651.

Křivan, V. 1996. Optimal foraging and predator-prey dynamics. Theoretical Population Biology 49:265-290.

Křivan, V. 1997. Dynamic ideal free distribution: Effects of optimal patch choice on predatorprey dynamics. American Naturalist 149:164-178.

Křivan, V. and A. Sikder. 1999. Optimal foraging and predator-prey dynamics II. Theoretical Population Biology 55:111-126.

Křivan, V. and J. Eisner. 2003. Optimal foraging and predator-prey dynamics III. Theoretical Population Biology 63:269-279. doi:10.1016/S0040-5809(03)00012-1. 
Křivan, V. and J. Eisner. 2006. The effect of the Holling type II functional response on apparent competition. Theoretical Population Biology 70:421-430.

Křivan, V., R. Cressman and C. Schneider. 2008. The Ideal Free Distribution: A review and synthesis of the game theoretic perspective. Theoretical Population Biology 73:403-425.

Loeuille, N. 2010. Consequences of adaptive foraging in diverse communities. Functional Ecology 24:18-27.

Martinez, N. D., R. J. Williams and J. A. Dunne. 2006. Diversity, complexity, and persistence in large model ecosystems. Pages 163-185 in M. Pascual and J. A. Dunne, eds. Ecological networks: linking structure to dynamics in food webs. Oxford University Press, Oxford, UK.

May, R. M. 1972. Will a large complex system be stable? Nature 238:413-414.

McCann, K., A. Hastings and G. R. Huxel. 1998. Weak trophic interactions and the balance of nature. Nature 395:794-798.

Milo, R., S. Shen-Orr, S. Itzkovitz, N. Kashtan, D. Chklovskii and U. Alon. 2002. Network motifs: Simple building blocks of complex networks. Science 298:824-827.

Murdoch, W. W. 1969. Switching in general predators: Experiments on predator specificity and stability of prey populations. Ecological Monographs 39:335-354.

Murdoch, W. W. and A. Oaten. 1975. Predation and population stability. Pages 1-131 in A. Macfadyen, ed. Advances in Ecological Research. Academic Press.

Petchey, O. L., A. P. Beckerman, J. O. Riede and P. H. Warren. 2008. Size, foraging, and food web structure. Proceedings of the National Academy of Sciences of the USA 105:41914196.

Ruxton, G. D. 1995. Short term refuge use and stability of predator-prey models. Theoretical Population Biology 47:1-17.

Stephens, D. W. and J. R. Krebs. 1986. Foraging theory. Princeton University Press, Princeton, NJ, USA. 
1 Tinbergen, N. 1960. The natural control of insects in pine woods: Vol. I. Factors influencing the intensity of predation by songbirds. Archives Neelandaises de Zoologie 13:265-343.

Uchida, S. and B. Drossel. 2007. Relation between complexity and stability in food webs with $4 \quad$ adaptive behavior. Journal of Theoretical Biology 247:713-722.

Uchida, S., B. Drossel and U. Brose. 2007. The structure of food webs with adaptive behaviour. Ecological Modelling 206:263-276.

Williams, R. J. and N. D. Martinez. 2000. Simple rules yield complex foodwebs. Nature $8 \quad 404: 180-183$.

Yodzis, P. and K. O. Winemiller. 1999. In search of operational trophospecies in a tropical aquatic food web. Oikos 87:327-340. 


\section{Food webs with slow adaptation}

A

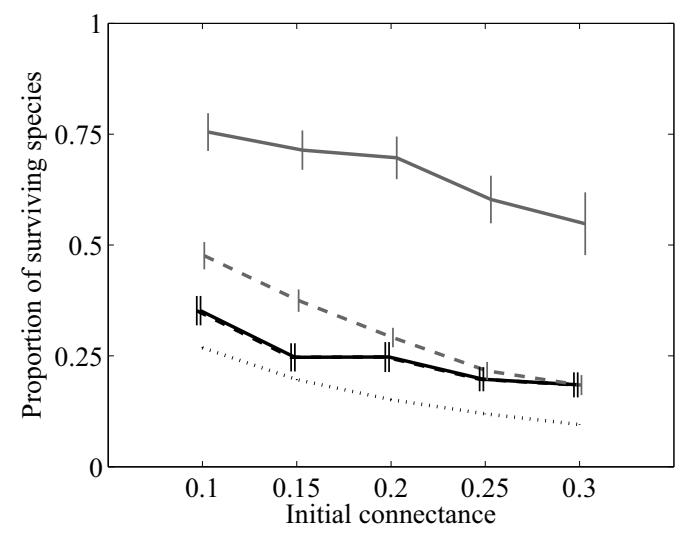

$\mathrm{C}$

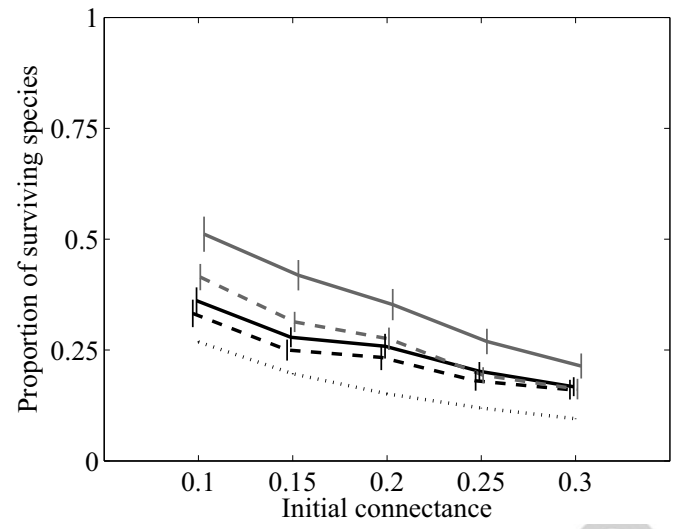

\section{Food webs with fast adaptation}

$\mathrm{B}$

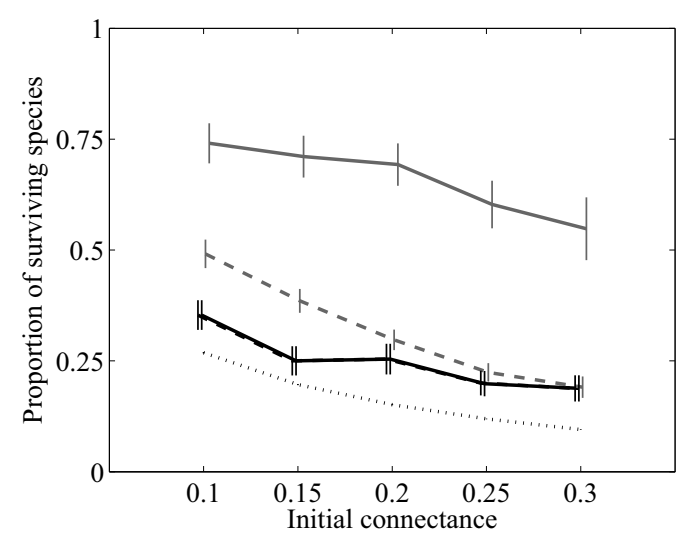

$\mathrm{D}$

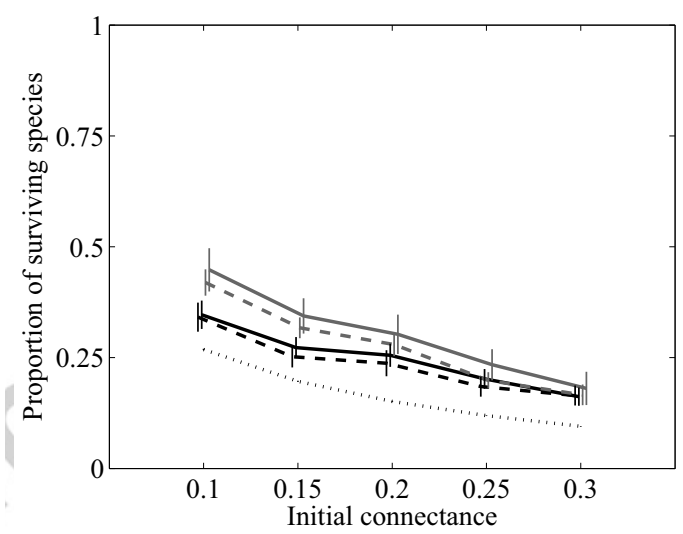

Figure 1: Food web dynamics: the proportion of surviving species as a function of the (initial) food web connectance. Panels: $\mathrm{A}-$ parameter set $1, \nu=0.25$; $\mathrm{B}$ - parameter set $1, \nu=4$; $\mathrm{C}-$ parameter set $2, \nu=0.25 ; \mathrm{D}$ - parameter set $2, \nu=4$. Other parameters: $S=30,50$ simulation runs. Legend: solid gray line - search image model with adaptation, dashed gray line - search image model without adaptation, solid black line - diet choice model with adaptation, dashed black line - diet choice model without adaptation, dotted black line - proportion of basal species as generated by the niche model. Vertical bars represent a measure of variability that allows for direct visual assessment of statistical significance of differences between any two scenarios simply put, any two scenarios whose vertical bars do not overlap differ significantly (Andrews et al. 1980). The five lines in each panel are slightly shifted in the horizontal direction so that all vertical bars are clearly visible. 


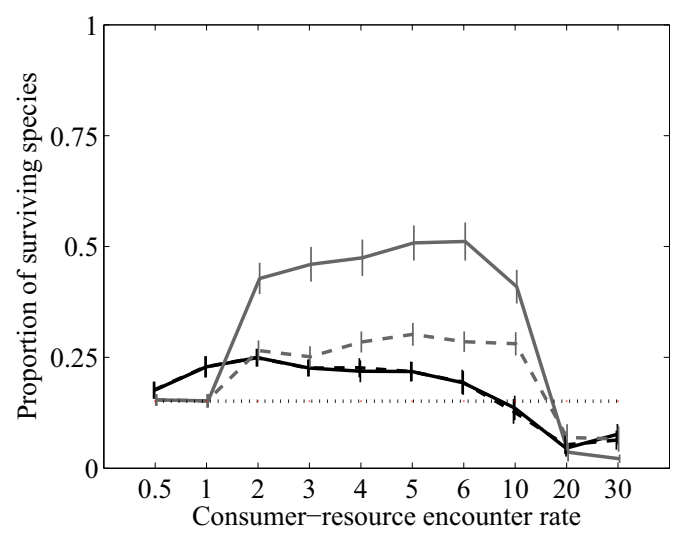

Figure 2: Food web dynamics: the proportion of surviving species as a function of the consumer-resource encounter rate $\lambda$. Other parameters: $S=30, C=0.2, \nu=0.25,50$ simulation runs, other parameters taken from the parameter set 1. Legend as in Fig. 1. 
A

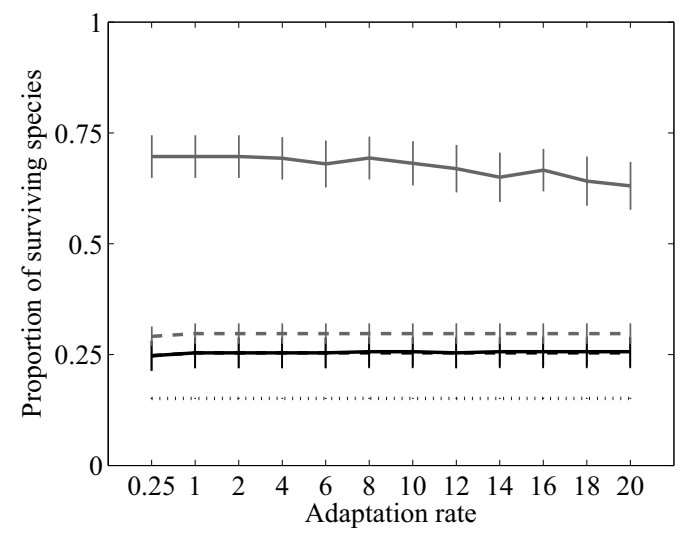

B

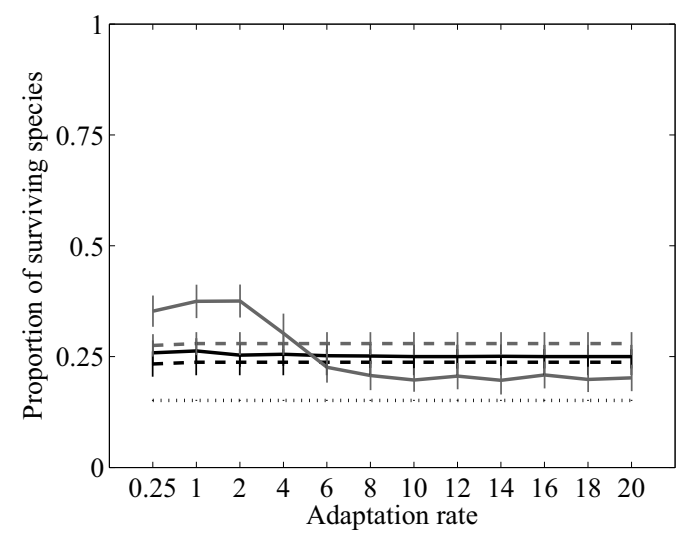

Figure 3: Food web dynamics: the proportion of surviving species as a function of the adaptation rate $\nu$. Other parameters: $S=30, C=0.2,50$ simulation runs, parameters taken from the parameter set 1 (A) or 2 (B). Legend as in Fig. 1. 
A

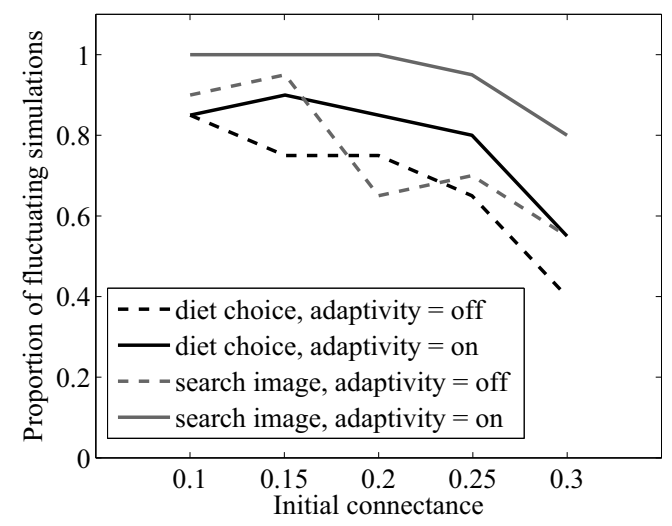

B

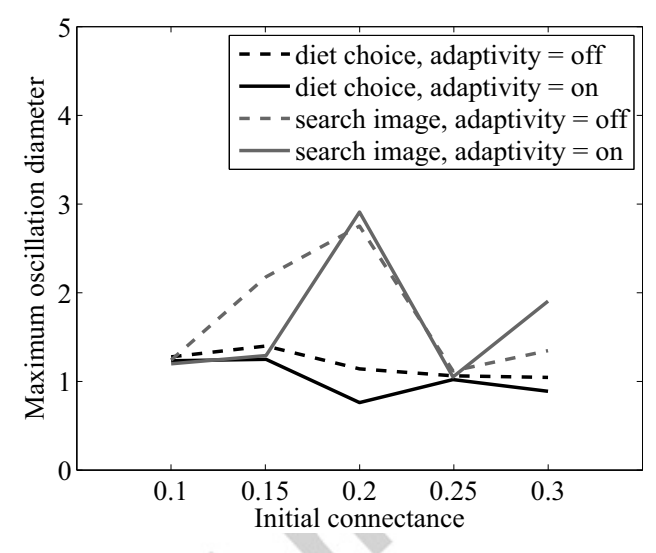

C

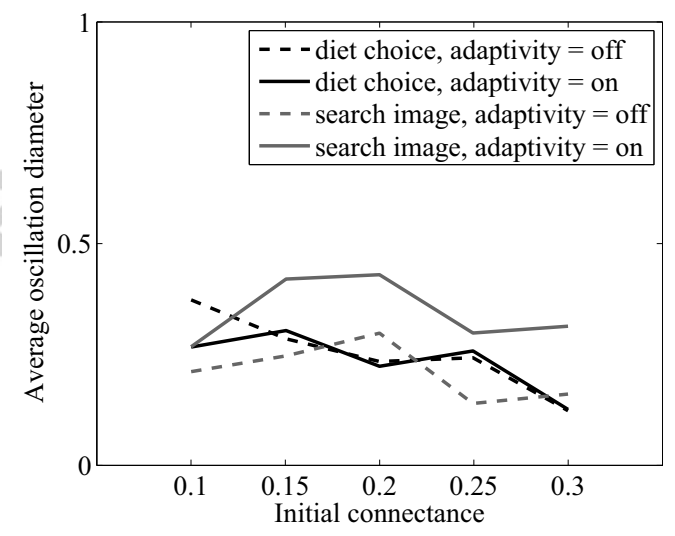

Figure 4: Some characteristics of population dynamics run on food webs consisting of 30 species and generated by the niche model; $\nu=0.25,50$ simulation runs, parameters taken from the parameter set 2 . 
A

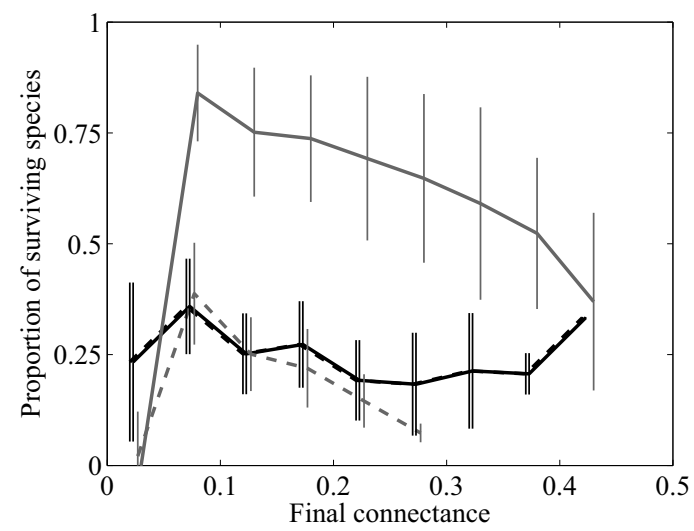

B

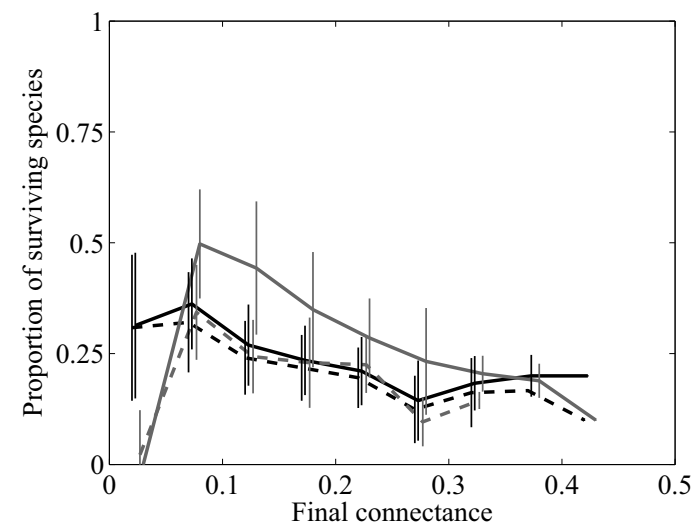

Figure 5: Food web dynamics: the proportion of surviving species correlated to the final food web connectance. Other parameters: $S=30, \nu=0.25,50$ simulation runs, parameters taken from the parameter set 1 (A) or 2 (B). Legend as in Fig. 1. The actual procedure of creating this figure is as follows. For each of the five examined values of (initial) $C$ and each of the four adaptive foraging model scenarios 50 simulation runs have been conducted. Ten bins were then created to which the proportions of surviving species corresponding to connectances in the ranges $0-0.05,0.05-0.1, \ldots, 0.95-1$ were put, and means and standard deviations were calculated within each bin and eventually plotted. 


\begin{tabular}{|c|c|c|c|}
\hline Parameter & Meaning & Set 1 & Set 2 \\
\hline$d_{i}$ & mortality rate of species $i$ (non-basal) & 0.5 & {$\left[\begin{array}{ll}0.3 & 0.7\end{array}\right]$} \\
\hline$r_{i}$ & intrinsic growth rate of species $i$ (basal) & 1.0 & {$\left[\begin{array}{lll}0.8 & 1.2\end{array}\right]$} \\
\hline$s_{i}$ & $\begin{array}{l}\text { measure of intraspecific competition of species } \\
i \text { (basal) }\end{array}$ & 1.0 & {$\left[\begin{array}{ll}0.8 & 1.2\end{array}\right]$} \\
\hline$\lambda_{i j}$ & encounter rate between species $i$ and $j$ & 6 & {$\left[\begin{array}{ll}4 & 8\end{array}\right]$} \\
\hline$e_{i j}$ & $\begin{array}{l}\text { conversion efficiency of species } i \text { when con- } \\
\text { sumed by species } j\end{array}$ & 0.5 & {$\left[\begin{array}{ll}0.2 & 0.8\end{array}\right]$} \\
\hline$h_{i j}$ & $\begin{array}{l}\text { handling time of species } i \text { when consumed by } \\
\text { species } j\end{array}$ & 0.4 & {$\left[\begin{array}{ll}0.2 & 0.6\end{array}\right]$} \\
\hline- & initial densities for basal species & & {$\left[\begin{array}{ll}0.1 & 1\end{array}\right]$} \\
\hline- & initial densities for non-basal species & {$\left[\begin{array}{ll}0.01 & 0.5\end{array}\right]$} & {$\left[\begin{array}{ll}0.01 & 0.5\end{array}\right]$} \\
\hline
\end{tabular}

Table 1: Model parameters, their meaning and values used in baseline simulations. Regarding parameter values, we specify intervals (set 2) or constant values (interval midpoints, set 1). Note that the initial conditions are always defined via intervals. Although we do not regard constant parameters identical to all species particularly realistic, we include this possibility in order to enable comparison of our results with those of other studies. 